\title{
Portfolio Allocation using Multivariate Variance Gamma
}

\author{
Asmerilda Hitaj and Lorenzo Mercuri
}

December 20, 2011

\begin{abstract}
In this paper we investigate empirically the effect of using higher moments in portfolio allocation when parametric and non parametric models are used. The non parametric model considered in this paper is the sample approach while the parametric one is constructed assuming Multivariate Variance Gamma (MVG henceforth) joint distribution for asset returns. We consider the MVG models proposed by Madan and Seneta (1990), Semeraro (2006) and Wang (2009).

We perform an out-of-sample analysis comparing the optimal portfolios obtained using the MVG models and the sample approach. Our portfolio is composed of 18 assets selected from the S\&P500 Index and the dataset consists in daily returns observed in the period ranging from $01 / 04 / 2000$ to $01 / 09 / 2011$.
\end{abstract}

\section{Introduction}

Since the seminal work of Markowitz 1952 [15], the relevance of moments in portfolio allocation has been recognized in financial literature (see [18]). However, owing to its simplicity, the mean-variance model does not take the investor's preferences for high skewness and low kurtosis into account (see [10], [2] and references therein).

This problem is relevant when the investor's utility function is not quadratic or the empirical joint distribution of financial returns is different from the multivariate normal, as is usual in real markets. As observed in [14], the short-time horizon is the most appropriate for professional investors who frequently rebalance their positions; in this case, returns seem to display higher level of kurtosis which cannot be modeled with normal distributions.

To overcome the inadequacy of mean-variance model, three complementary approaches have been proposed introducing higher moments in portfolio allocation. The first is to construct an efficient frontier by incorporating higher moments (see [8], [2] and [3]). The second is to model asset returns distribution using some approximate densities like Gram-Chalier expansion, Edgeworth series or Hermite polynomials (see [6] and references therein). The last is based 
on approximating the utility function through the Taylor series, writing the expected utility as linear combination of the portfolio's central moments where the coefficients depend on the investor's risk aversion (see [10] and references therein).

When Taylor approximation is used, the estimation problem of the moments and comoments is crucial. Several approaches have been proposed, for the estimation of these, that can be classified in non-parametric and parametric (see [17]). The first class, based on the law of large numbers, performs well when the number of observations, in stationary time series, is very large. In this case the non-parametric estimators are very flexible and are not influenced by the true distribution of returns. The most famous and used estimators are the sample comoments. These estimators are characterized by high estimation error when the number of observations is small. To overcome this problem, two methodologies have been proposed: the first is using improved estimators (see [16] and recently [7]) and the second is assuming a particular joint distribution of returns (see [9] and the references therein for a general survey).

In this paper we follow the second approach, where we assume a Multivariate Variance Gamma distribution for log-returns.

In the univariate case, the variance gamma density is a normal variance-mean mixture with gamma mixing density and, in a static period, Madan and Seneta [13] have shown its ability to capture the stylized facts. The extension to the multivariate case is not as simple as in the multivariate normal distribution. Several generalizations have been proposed. In this paper, we focus our attention on MVG introduced by [13] for the symmetric case (see [5] and the references therein for generalization), the Semeraro model (see [19]) and the Wang model (see [20]).

The first model is the simplest way to construct a multivariate variance gamma starting from a multivariate normal distribution, since it is a multivariate normal variance-mean mixture with a common univariate mixing gamma density. The model, however, does not allow the independence case and the sharing of the same mixing density presents some problems when we want to fit the sample covariance matrix once the marginals are fixed.

To overcome these limits, Semeraro (see [19]) proposed a mixture of independent multivariate normals in which the mixing random variable has a multivariate gamma distribution. The dependence is introduced considering a multivariate dependent random vector. In this model, the parameters, which control the dependence, can be fitted using the sample covariance. Although the model includes the independence between assets and the estimation procedure is easily implemented, Wang [20] observed that this model is not flexible in capturing different dependence structures. For this reason, he proposed a multivariate model based on the property of the sum of independent variance gamma.

Our aim is to investigate the impact of higher moments on the investor's terminal wealth, under the assumption of MVG distributions for log returns and compare it with the sample approach. Our empirical analysis is carried on a portfolio composed by 18 assets selected from the S\&P500 Index and the dataset consists in daily returns observed in the period ranging from 01/04/2000 
to $01 / 09 / 2011$. We perform an out-of-sample comparison between the Multivariate Variance Gamma models and the sample approach (see recently [7] and references therein) in terms of Monetary Utility Gain/Loss as introduced by Ang and Bekaert [1].

The paper is organized as follows. In Section 2 we explain the investor's problem and how to introduce the higher moments in portfolio allocation. In Section 3 we review the MVG models used for the portfolio allocation, compute comoments and the analytical form for CARA expected utility functions. Section 4 is dedicated to the empirical analysis and Section 5 to conclusions.

\section{Portfolio Allocation}

In this section, we review how the investor's problem can be easily solved using the Taylor series expansion (see [10]) in a static period. We consider an agent, with utility function $u(W)$, whose objective is to maximize the expected utility of wealth:

$$
\left\{\begin{array}{c}
\max _{w_{i}} E\left[u\left(\sum_{i=1}^{N} w_{i} X_{i}\right)\right] \\
\text { s.t. } \\
\sum_{i=1}^{N} w_{i}=1 \\
l b \leq w_{i} \leq u b \text { for } i=1, \ldots, N
\end{array}\right.
$$

where $N$ is the number of risky assets, $w:=\left(w_{1}, \ldots, w_{N}\right)$ is the fraction of wealth that the investor allocates in the available risky assets, $l b$ and $u b$ are, respectively, lower and upper bounds of the weights when applicable and $X:=$ $\left(X_{1}, \ldots, X_{N}\right)$ is the vector of returns.

Problem 1 cannot always be solved directly since we would need an analytical expression for the expected utility. To overcome this limit, a Taylor expansion has been used in literature (see [10] and references therein).

Under the assumption that the joint moment generating function of a random vector $X$ exists and the function $u(W)$ satisfies some mild conditions [11], the expected utility can be written as:

$$
E[u(W)]=\sum_{j=0}^{+\infty} u^{(j)}(E[X]) \frac{E\left[(X-E(X))^{j}\right]}{j !},
$$

where $u^{(j)}$ is the $j^{\text {th }}$ derivative of the utility function at the point $E(X)$.

In literature there exist different types of utility functions, like the exponential, power, logarithmic etc.

In this paper, we focus our attention on the exponential utility function with a constant absolute risk aversion coefficient, $\lambda$. In this case, considering the fourth order Taylor expansion, formula 2 can be written as follows:

$$
E[u(W)] \approx-e^{-\lambda\left(\mu_{p}\right)}\left[1+\frac{1}{2} \lambda^{2} \sigma_{p}^{2}-\frac{1}{6} \lambda^{3} s_{p}^{3}+\frac{1}{24} \lambda^{4} k_{p}^{4}\right],
$$


where $\mu_{p}, \sigma_{p}^{2}, s_{p}^{3}, k_{p}^{4}$ are respectively the mean, variance, skewness and kurtosis of the portfolio, given by:

$$
\begin{aligned}
\mu_{p} & =\sum_{i=1}^{N} \mu_{i} w_{i}^{\prime}=\mu w^{\prime} \\
\sigma_{p}^{2} & =w M_{2} w^{\prime} \\
s_{p}^{3} & =w M_{3}\left(w^{\prime} \otimes w^{\prime}\right) \\
k_{p}^{4} & =w M_{4}\left(w^{\prime} \otimes w^{\prime} \otimes w^{\prime}\right),
\end{aligned}
$$

where $M_{2}, M_{3}$ and $M_{4}$ are the covariance, coskewness and cokurtosis matrices, respectively and $\otimes$ is the Kronecker product.

The investor's problem (1) becomes:

$$
\left\{\begin{array}{l}
\max _{w_{i}}-e^{-\lambda\left(\mu w^{\prime}\right)}\left[1+\frac{\lambda^{2}}{2} w M_{2} \quad w^{\prime}-\frac{\lambda^{3}}{6} w M_{3}\left(w^{\prime} \otimes w^{\prime}\right)+\frac{\lambda^{4}}{24} w M_{4}\left(w^{\prime} \otimes w^{\prime} \otimes w^{\prime}\right)\right] \\
\text { s.t } \sum_{i=1}^{N} w_{i}=1 \\
0 \leq w_{i} \leq 1
\end{array}\right.
$$

To find the optimal weights, we need to estimate the co-moments. The easiest way is to consider the sample estimators.

The sample mean of asset $i$ is:

$$
\widehat{\mu_{i}}=\frac{1}{T} \sum_{t=1}^{T} X_{i, t}
$$

where $T$ is the sample size.

The sample covariance between assets $i$ and $j$ is:

$$
\widehat{\sigma_{i j}^{2}}=\frac{\sum_{t=1}^{T}\left(X_{i t}-\widehat{\mu_{i}}\right)\left(X_{j t}-\widehat{\mu_{j}}\right)}{(T-1)}
$$

The sample coskewness between assets $i, j$ and $k$ is:

$$
\widehat{s_{i j k}}=\frac{T}{(T-1)(T-2)} \sum_{t=1}^{T}\left(X_{i t}-\widehat{\mu_{i}}\right)\left(X_{j t}-\widehat{\mu_{j}}\right)\left(X_{k t}-\widehat{\mu_{k}}\right) .
$$

The sample cokurtosis between assets $i, j, k$ and $l$ is:

$$
\widehat{k_{i j k l}}=\frac{T}{(T-2)(T-3)} \sum_{t=1}^{T}\left(X_{i t}-\widehat{\mu_{i}}\right)\left(X_{j t}-\widehat{\mu_{j}}\right)\left(X_{k t}-\widehat{\mu_{k}}\right)\left(X_{l t}-\widehat{\mu_{l}}\right) .
$$

\section{Multivariate Variance Gamma}

In this section, we analyze the three multivariate variance gamma models mentioned earlier: the MVG with univariate mixing density, the Semeraro model, 
where the dependence structure is obtained through a multivariate mixing density, and the Wang model that is built using the sum property of two independent variance gamma.

For each model, we investigate an estimation procedure based on two steps. In the first step, we obtain some parameters from the single time series, using the EM-algorithm proposed in Loregian et al. 2011 [12]. In the second one, we fix the remaining parameters by matching the sample and theoretical covariance matrices.

We derive closed-form formulas for moments and co-moments necessary to solve problem 4. For CARA utility functions, we obtain the analytical formula for expected utility. In this way, in the empirical analysis, we compare the outof-sample portfolios obtained with Taylor's approximation and the analytical expected utility.

\subsection{Univariate variance gamma model}

In this subsection, we review quickly the definition and the main features of the variance gamma model introduced in Finance by [13] (1990). The variance gamma distribution belongs to the family of normal variance mean mixtures and corresponds to the case of gamma mixing density.

A continuous random variable $X$ is variance gamma distributed if it can be written as:

$$
X:=\mu_{0}+\theta V+\sigma \sqrt{V} Z
$$

where $\mu_{0}, \theta \in \Re, \sigma \in[0, \infty[, Z \sim \mathbf{N}(0,1)$ and $V$ is a gamma random variable independent from $Z$.

In general, a gamma random variable $Y \sim \Gamma(\alpha, \beta)$ is identified univocally by two parameters, respectively shape parameter and the scale parameter. In order to identify a distribution as a variance gamma we need some constraints on the scale parameter: in some literature $\beta=\alpha$, so that $E[Y]=1$.

In this paper, we follow a different parametrization and fix $\beta=1$ meaning that $V \sim \Gamma(\alpha, 1)$.

The simplest way to have the representation of the density is by using the modified Bessel function of the third kind, see Madan et al. (1990) and references therein, where the m.g.f. is given by:

$$
M_{X}(c)=\exp \left(c \mu_{0}\right)\left(\frac{1}{1-c \theta-\frac{c^{2} \sigma^{2}}{2}}\right)^{\alpha}
$$

It is possible to show that the first four moments of a VG are:

$$
\begin{aligned}
E[X] & =\mu_{0}+\alpha \theta \\
E\left[(X-E[X])^{2}\right] & =\alpha\left(\theta^{2}+\sigma^{2}\right) \\
E\left[(X-E[X])^{3}\right] & =\alpha \theta\left(3 \sigma^{2}+2 \theta^{2}\right) \\
E\left[(X-E[X])^{4}\right] & =3\left(\alpha^{2}+\alpha\right) \sigma^{4}+\left(\alpha^{2}+2 \alpha\right)\left(6 \theta^{2} \sigma^{2}+3 \theta^{4}\right) .
\end{aligned}
$$


As shown by Madan and Seneta [13] this distribution seems to have a good ability to fit the historical log return distribution of a general asset and is popular for option pricing ${ }^{1}$.

In the following subsection we will explain three methods to generalize an univariate variance gamma to a multivariate variance gamma.

\subsection{Multivariate variance gamma with a common gamma mixing density}

The simplest way to generalize the univariate variance gamma distribution is to consider a multivariate normal variance mean mixture with a common univariate gamma mixing density.

In this model the random vector $X$ is defined as:

$$
X=\mu+\theta V+\sqrt{V} \Sigma^{1 / 2} Z
$$

where $Z \sim \mathbf{N}\left(0, I_{N}\right)$ is composed by $N$ i.i.d standard normals, $V \sim \Gamma(\alpha, 1)$, is a univariate gamma independent of $Z, \mu \in \mathbf{R}^{\mathbf{N}}, \theta \in \mathbf{R}^{\mathbf{N}}$, and $\Sigma^{1 / 2}$ is a lower triangular matrix:

$$
\Sigma_{i j}^{1 / 2}=\left\{\begin{array}{l}
a_{i j} \text { if } i \geq j \\
0 \text { otherwise }
\end{array} .\right.
$$

The $i^{\text {th }}$ component of the random vector $X$ is a univariate variance gamma model, given as:

$$
X_{i}=\mu_{i}+\theta_{i} V+\sqrt{V} \sum_{h=1}^{i} a_{i h} Z_{h}
$$

The multivariate variance gamma with common mixing random variable, as the univariate case, does not have a simple form for the density probability function. When we consider a large number of assets (this is the case of portfolio allocation) the estimation procedure based on the joint Likelihood function has many problems (see [4] and references therein). For this reason we consider an estimation procedure that can be summarized in the following steps:

1) For each financial time series, we estimate the marginal parameters, $\mu_{i}, \theta_{i}, \sigma_{i}^{2}$ and $\alpha_{i}$ by the Maximum Likelihood procedure.

2) Since, in this model, the shape parameter $\alpha$ must be the same for all assets we fix it using the sample mean: $\hat{\alpha}=\frac{1}{N} \sum_{i=1}^{N} \alpha_{i}$.

3) Having fixed $\alpha$ we reestimate $\mu_{i}, \theta_{i}, \sigma_{i}^{2}$ using MLE procedure asset by asset.

4) To estimate all the parameters in $\Sigma^{1 / 2}$ we minimize the Frobenius norm between the sample covariance matrix and the theoretical given by $\Sigma=$ $\Sigma^{1 / 2} *\left(\Sigma^{1 / 2}\right)^{\prime}$ under the constraints: $\sigma_{i}^{2}=\sum_{h=1}^{i} a_{i h}^{2}$ for $i=1, \ldots, N$.

\footnotetext{
${ }^{1}$ The variance gamma model is implemented in Bloomberg
} 
The moments and co-moments, in this model, can be derived through straightforward calculation.

The mean of the $i^{\text {th }}$ component is:

$$
E\left(X_{i}\right)=\mu_{i}+\theta_{i} \alpha
$$

The elements of the covariance matrix are:

$$
\begin{cases}\operatorname{Var}\left(X_{i}\right)=\alpha\left(\theta_{i}^{2}+\sigma_{i}^{2}\right) & \text { for } i=j \\ \operatorname{Cov}\left(X_{i}, X_{j}\right)=\alpha\left(\theta_{i} \theta_{j}+\sigma_{i j}\right) & \text { for } i \neq j\end{cases}
$$

where $\sigma_{i j}=\sum_{h=1}^{\min (i, j)} a_{i h} a_{j h}$.

The elements of the coskewness matrix are the following:

$$
\begin{cases}s_{i i i}=\alpha \theta_{i}\left(3 \sigma_{i}^{2}+2 \theta_{i}^{2}\right) & \text { for } i=j=k \\ s_{i i k}=\alpha\left(\theta_{k} \sigma_{i}^{2}+2 \theta_{i} \sigma_{i k}+2 \theta_{k} \theta_{i}^{2}\right) & \text { for } i=j \neq k \\ s_{i j k}=\alpha\left(\theta_{k} \sigma_{i j}+\theta_{i} \sigma_{j k}+\theta_{j} \sigma_{i k}+2 \theta_{k} \theta_{i} \theta_{j}\right) & \text { for } i \neq j \neq k\end{cases}
$$

The elements of the cokurtosis are:

$$
\begin{array}{rlrl}
k_{i i i i}= & 3\left(\alpha^{2}+\alpha\right) \sum_{h=1}^{i} a_{i h}^{4}+ & & \text { for } i=j=k=l \\
& +\left(\alpha^{2}+2 \alpha\right)\left(6 \theta_{i}^{2} \sigma_{i}^{2}+3 \theta_{i}^{4}\right) & & \\
k_{i i i l}= & 3\left(\alpha^{2}+\alpha\right) \sum_{h=1}^{\min (i, l)} a_{i h}^{3} a_{l h}+ & & \text { for } i=j=k \neq l \\
& +\left(\alpha^{2}+2 \alpha\right)\left(3 \theta_{i} \theta_{l} \sigma_{i}^{2}+3 \theta_{i}^{2} \sigma_{i l}+3 \theta_{i}^{3} \theta_{l}\right) & & \\
k_{i i l l}= & 3\left(\alpha^{2}+\alpha\right) \sum_{h=1}^{\min (i, l)} a_{i h}^{2} a_{l h}^{2}+ & & \\
& +\left(\alpha^{2}+2 \alpha\right)\left(\theta_{l}^{2} \sigma_{i}^{2}+4 \theta_{i} \theta_{l} \sigma_{i l}+\theta_{i}^{2} \sigma_{l}^{2}\right)+ & & \\
& +\left(3 \alpha^{2}+6 \alpha\right) \theta_{i}^{2} \theta_{l}^{2} & & \\
k_{i i k l}= & 3\left(\alpha^{2}+\alpha\right) \sum_{h=1}^{\min (i, k, l)} a_{i h}^{2} a_{k h} a_{l h}+ & \\
& +\left(\alpha^{2}+2 \alpha\right)\left(\theta_{k} \theta_{l} \sigma_{i}^{2}+2 \theta_{i} \theta_{l} \sigma_{i k}+2 \theta_{i} \theta_{k} \sigma_{i l}+\theta_{i}^{2} \sigma_{k l}\right)+l \\
& +\left(3 \alpha^{2}+6 \alpha\right) \theta_{i}^{2} \theta_{k} \theta_{l} & & \\
k_{i j k l}= & 3\left(\alpha^{2}+\alpha\right) \sum_{h=1}^{\min (i, j, k, l)} a_{i h} a_{j h} a_{k h} a_{l h}+ & \\
& +\left(\alpha^{2}+2 \alpha\right)\left(3 \theta_{i} \theta_{j} \theta_{k} \theta_{l}+\theta_{i} \theta_{l} \sigma_{j k}+\theta_{i} \theta_{k} \sigma_{j l}+\theta_{i} \theta_{j} \sigma_{k l}\right)+ & & \text { for } i \neq j \neq k \neq l \\
& +\left(\alpha^{2}+2 \alpha\right)\left(\theta_{k} \theta_{l} \sigma_{i j}+\theta_{j} \theta_{l} \sigma_{i k}+\theta_{j} \theta_{k} \sigma_{i l}\right) &
\end{array}
$$

Using the joint characteristic function we derive an analytical formula for the expected utility when the investor's preferences are represented by a CARA 
function:

$$
\begin{aligned}
U\left(\sum_{i=1}^{N} w_{i} X_{i}\right) & =E\left(-\exp \left(-\lambda \sum_{i=1}^{N} w_{i} X_{i}\right)\right) \\
& =-\exp \left(-\lambda \sum_{i=1}^{N} w_{i} \mu_{i}\right) * \\
& * \exp \left(\alpha \ln \left(\frac{1}{1+\lambda \sum_{i=1}^{N} w_{i} \theta_{i}-\frac{\lambda^{2}}{2} \sum_{i, j=1}^{N} w_{i} w_{j} \sum_{h=1}^{\min (i, j)} a_{i h} a_{j h}}\right)\right)
\end{aligned}
$$

\subsection{Semeraro model}

The main drawback of the multivariate variance gamma model illustrated in the previous subsection is the sharing of the same parameter $\alpha$ which puts a heavy restriction to the joint distribution. Independence between components is not satisfied and the considered historical estimation procedure is based on the trick of fixing $\hat{\alpha}$ to the sample mean.

To overcome this problem, Semeraro proposed a different multivariate variance gamma distribution where each component of the mixing random vector is gamma distributed.

The random vector $X$ follows the multivariate variance gamma model proposed by Semeraro in 2006 if the $i^{\text {th }}$ component is defined as:

$$
X_{i}=\mu_{i}+\theta_{i} G_{i}+\sigma_{i} \sqrt{G_{i}} W_{i} \text { for } i=1, \ldots, N
$$

where $W_{1} \ldots W_{N}$ are independent standard normals and $G_{i}$ is a random variable defined as:

$$
G_{i}=Y_{i}+a_{i} Z \text { for } i=1, \ldots, N \text { and } a_{i} \geq 0
$$

where $Y_{i} \sim \Gamma\left(l_{i}, m_{i}\right)$ and $Z \sim \Gamma(n, k) . Y_{i}, Z$ and $W_{i}$ are independent from each other.

To ensure that $X_{i}$ is variance gamma distributed we require that $G_{i}$ follows a gamma distribution. This is true under two alternative constraints: $a_{i}$ is equal to zero, that corresponds to the independence case, or $a_{i}=k / m_{i}>0$. The last case, from the summation property of two independent gamma with the same scale parameter, implies $G_{i} \sim \Gamma\left(l_{i}+n ; m_{i}\right)$.

Although we can build a multivariate distribution with general values for $a_{i}$ parameters, in this paper we focus our attention only on the dependence between assets that corresponds to $a_{i}=k / m_{i}$ case.

The total number of parameters in this model is $5 N+2$ where $N$ is the number of assets. The estimation approach is composed of two steps:

1) For each time series we estimate the marginal distribution maximizing the log-likelihood function. Therefore from the marginal distribution we obtain the estimators $\hat{\mu}_{i}, \hat{\theta}_{i}, \hat{\sigma}_{i}, \hat{l}_{i}$, where $\hat{\theta}_{i}=\frac{\theta_{i}}{m_{i}}, \hat{l}_{i}=l_{i}+n$ and $\hat{\sigma}_{i}=$ $\frac{\sigma_{i}}{\sqrt{m_{i}}}$. 
2) In the second step we estimate $n$, minimizing the Frobenius distance between the theoretical covariance matrix and the empirical one. Since the theoretical variance for each asset is known after we have completed the first step, the minimization problem is equivalent to the following:

$$
\min _{n} \sum_{\substack{i, j=1 \\ i \neq j}}^{N}\left(\hat{\theta}_{i} \hat{\theta}_{j} n-\operatorname{cov}_{\text {sample }}\left(X_{i}, X_{j}\right)\right)^{2} .
$$

Observe that in order to match the theoretical covariance with the sample one we have only one free parameter $(n)$. This can be a problem when the number of assets in our portfolio is high.

Now we derive analytical formulas for moments and comoments. For the $i^{\text {th }}$ component of the mean vector, we have:

$$
E\left(X_{i}\right)=\mu_{i}+\frac{\theta_{i}}{m_{i}}\left(l_{i}+n\right) .
$$

The components of the covariance matrix are given by

$$
\begin{cases}\operatorname{Var}\left(X_{i}\right)=\left(\frac{\theta_{i}^{2}}{m_{i}^{2}}+\frac{\sigma_{i}^{2}}{m_{i}}\right)\left(l_{i}+n\right) & \text { for } i=j \text { (diagonal elements) } \\ \operatorname{Cov}\left(X_{i}, X_{j}\right)=\frac{\theta_{i}}{m_{i}} \frac{\theta_{j}}{m_{j}} n & \text { for } i \neq j \text { (off-diagonal elements) }\end{cases}
$$

The formulas for the elements of coskewness matrix are:

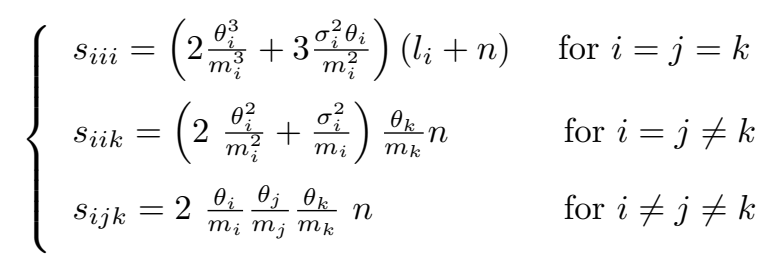


The elements of cokurtosis matrix are given by:

$$
\left\{\begin{aligned}
k_{i i i i}= & \frac{\theta_{i}^{4}}{m_{i}^{4}}\left[3 l_{i}^{2}+6 l_{i}+6 l_{i} n+3 n^{2}+6 n\right]+ & & \\
& +6 \frac{\theta_{i}^{2} \sigma_{i}^{2}}{m_{i}^{3}}\left[l_{i}\left(l_{i}+2\right)+n(n+2)+2 l_{i} n\right]+ & & \text { for } i=j=k=l \\
& +3 \frac{\sigma_{i}^{4}}{m_{i}^{2}}\left[\left(l_{i}+1\right) l_{i}+(n+1) n+2 l_{i} n\right] & & \\
k_{i i i l}= & 3 \frac{\theta_{i}^{3}}{m_{i}^{3}} \frac{\theta_{l}}{m_{l}} l_{i} n+3 \frac{\theta_{i}}{m_{i}} \frac{\theta_{l}}{m_{l}} \frac{\sigma_{i}^{2}}{m_{i}} n(n+2)+ & & \text { for } i=j=k \neq l \\
& +3 \frac{\theta_{i}}{m_{i}} \frac{\sigma_{i}^{2}}{m_{i}} \frac{\theta_{l}}{m_{l}} l_{i} n+\frac{\theta_{i}^{3}}{m_{i}^{3}} \frac{\theta_{l}}{m_{l}}\left(3+\frac{6}{n}\right) n^{2} & & \\
k_{i i l l}= & \frac{\theta_{i}^{2}}{m_{i}^{2}} \frac{\theta_{l}^{2}}{m_{l}^{2}} l_{i}\left[l_{l}+n\right]+\frac{\theta_{i}^{2}}{m_{i}^{2}} \frac{\theta_{l}^{2}}{m_{l}^{2}}\left[l_{l} n+\left(3+\frac{6}{n}\right) n^{2}\right]+ & & \\
& +\frac{\theta_{i}^{2}}{m_{i}^{2}} \frac{\sigma_{l}^{2}}{m_{l}} l_{i}\left[l_{l}+n\right]+\frac{\theta_{i}^{2}}{m_{i}^{2}} \frac{\sigma_{l}^{2}}{m_{l}}\left[n l_{l}+n(n+2)\right]+ & & \text { for } i=j \neq k=l \\
& +\frac{\theta_{l}^{2}}{m_{l}^{2}} \frac{\sigma_{i}^{2}}{m_{i}} l_{l}\left[l_{i}+n\right]+\frac{\theta_{l}^{2}}{m_{l}^{2}} \frac{\sigma_{i}^{2}}{m_{i}}\left[n l_{i}+n(n+2)\right] & & \\
k_{i i k l}= & n \frac{\theta_{i}^{2}}{m_{i}^{2}} \frac{\theta_{k}}{m_{k}} \frac{\theta_{l}}{m_{l}}\left[l_{i}+\left(3+\frac{6}{n}\right) n\right]+ & & \\
& +n \frac{\sigma_{i}^{2}}{m_{i}} \frac{\theta_{k}}{m_{k}} \frac{\theta_{l}}{m_{l}}\left[l_{i}+(n+2)\right] & & \text { for } i=j \neq k \neq l \\
k_{i j k l}= & \frac{\theta_{i}}{m_{j}} \frac{\theta_{j}}{m_{j}} \frac{\theta_{k}}{m_{k}} \frac{\theta_{l}}{m_{l}}\left(3+\frac{6}{n}\right) n^{2} & & \text { for } i \neq j \neq k \neq l
\end{aligned}\right.
$$

For this model, the analytical expression of the expected CARA utility function is:

$$
\begin{aligned}
E\left(u\left(\sum_{i=1}^{N} w_{i} X_{i}\right)\right. & =E\left(-\exp \left(-\lambda \sum_{i=1}^{N} w_{i} X_{i}\right)\right) \\
& =-\exp \left(-\lambda \sum_{i=1}^{N} w_{i} \mu_{i}\right) * \\
& * \exp \left(\sum_{i=1}^{N}\left(l_{i}\right) \ln \left(\frac{1}{1-\frac{1}{m_{i}}\left(\frac{\lambda^{2}}{2} w_{i}^{2} \sigma_{i}^{2}-w_{i} \theta_{i} \lambda\right)}\right)\right) * \\
& * \exp \left(n \ln \left(\frac{1}{1-\sum_{i=1}^{N} \frac{1}{m_{i}\left(\frac{\lambda^{2}}{2} w_{i}^{2} \sigma_{i}^{2}-w_{i} \theta_{i} \lambda\right)}}\right)\right) .
\end{aligned}
$$

\subsection{Wang model}

Wang (2009) proposed a different multivariate variance gamma model to overcome the limits of the first two MVG models previously studied. 
In order to introduce the Wang model, we recall the summation property of two independent VG random variables.

Proposition 1. Let $Y$ and $A$ be two independent $V G$ defined as:

$$
\begin{aligned}
& Y=\theta G+\sigma \sqrt{G} W_{Y} \\
& A=\theta V+\sigma \sqrt{V} W_{A}
\end{aligned}
$$

where $W_{Y}$ and $W_{A}$ are two independent standard normal variates and $G \sim$ $\Gamma\left(\alpha_{G}, 1\right)$ independent from $V \sim \Gamma\left(\alpha_{V}, 1\right)$. The random variable $X=Y+A$ is a VG with parameters $\left(\theta, \sigma, \alpha_{G}+\alpha_{V}\right)$.

A random vector $X$ follows a multivariate Wang distribution if each component can be expressed as:

$$
X_{i}=\mu_{i 0}+A_{i}+Y_{i} \text { for } i=1, \ldots, N
$$

where $Y_{i}, Y_{j}$ and $A_{i}$ are independent for $i, j=1, . ., N(i \neq j)$ and are defined as:

$$
\begin{aligned}
Y_{i} & =\theta_{i} G_{i}+\sigma_{G_{i}} \sqrt{G_{i}} W_{i}^{Y} \\
A_{i} & =\theta_{i} V+\sqrt{V} D_{i}
\end{aligned}
$$

where $D_{i}=\sum_{h=1}^{i} a_{i h} W_{h}^{A}$ for $i=1, \ldots, N . W_{i}^{Y} \sim N(0,1), W_{i}^{A} \sim N(0,1)$, $V \sim \Gamma\left(\alpha_{V}, 1\right)$ and $G_{i} \sim \Gamma\left(\alpha_{G_{i}}, 1\right)$ are independent from each other.

We observe that the $D_{i}$ are normal random variables correlated and $E\left(D_{i}\right)=0$ and $\operatorname{var}\left(D_{i}\right)=\sum_{h=1}^{i} a_{i h}^{2}$ :

$$
D_{i} \stackrel{d}{=} \sqrt{\sum_{h=1}^{i} a_{i h}^{2} Z_{i}}
$$

for $i=1, . ., N, Z_{i}$ are correlated standard normals.

To ensure that the random vector $X$ is a multivariate variance gamma we require $\sigma_{i}^{2}:=\sigma_{G_{i}}^{2}=\sum_{h=1}^{i} a_{i h}^{2}$. Therefore, the marginal distribution of the $i^{\text {th }}$ component of the random vector $X$ is a variance gamma with parameters $\left(\mu_{i 0}, \theta_{i}, \sigma_{i}, \alpha_{i}=\alpha_{V}+\alpha_{G_{i}}\right)$.

The number of parameters that we need to estimate in this model is $\frac{N^{2}+7 N+2}{2}$ and, as in the previous section, we use a two-step algorithm for their estimation. Our procedure can be summarized as follows:

1) First we estimate the marginal parameters $\mu_{i 0}, \theta_{i}, \sigma_{i}, \alpha_{i}=\alpha_{V}+\alpha_{G_{i}}$ using the MLE on each time series.

2) Having fixed the marginal parameters, we estimate $\alpha_{V}, \alpha_{G_{i}}$ and $a_{h \leq i}$ for $i=1, \ldots, N$, by matching the theoretical and the sample covariances. We observe that the number of parameters to estimate is $\frac{N *(N+1)}{2}+1$ and the number of constraints arising from the elements of the sample covariance 
matrix is $\frac{N(N+1)}{2}$. As proposed in [20], we fix $\alpha_{V}=\min \left(\alpha_{i}\right)$. An alternative way is to choose $\alpha_{V}$ such that it minimizes the distance between the sample and theoretical coskewness.

We derive analytical formulas for moments and co-moments. The elements of the mean vector are given by:

$$
E\left(X_{i}\right)=\mu_{i 0}+\theta_{i}\left(\alpha_{v}+\alpha_{G_{i}}\right) .
$$

The equations obtained for the calculation of all elements of the theoretical covariance matrix are:

$$
\left\{\begin{array}{lc}
\operatorname{Var}\left(X_{i}\right)=\left(\theta_{i}^{2}+\sigma_{i}^{2}\right)\left(\alpha_{V}+\alpha_{G_{i}}\right) & \text { for } i=j \text { (diagonal elements) } \\
\operatorname{Cov}\left(X_{i}, X_{j}\right)=\left(\theta_{i} \theta_{j}+\sigma_{i j}\right) \alpha_{V} & \text { for } i \neq j \text { (off-diagonal elements) }
\end{array}\right.
$$

The elements of coskewness matrix are calculated using the following formulas:

$$
\begin{cases}s_{i i i}=\left(3 \theta_{i} \sigma_{i}^{2}+2 \theta_{i}^{3}\right)\left(\alpha_{v}+\alpha_{G_{i}}\right) & \text { for } i=j=k \\ s_{i i k}=\alpha_{v}\left(\theta_{k} \sigma_{i}^{2}+2 \theta_{i} \sigma_{i k}+2 \theta_{k} \theta_{i}^{2}\right) & \text { for } i=j \neq k \\ s_{i j k}=\alpha_{v}\left(\theta_{k} \sigma_{i j}+\theta_{i} \sigma_{j k}+\theta_{j} \sigma_{i k}+2 \theta_{k} \theta_{i} \theta_{j}\right) & \text { for } i \neq j \neq k\end{cases}
$$

Each component of the cokurtosis matrix is calculated as follows:

$$
\begin{aligned}
& k_{i i i i}= 3\left(\alpha_{v}^{2}+\alpha_{G_{i}}^{2}+\alpha_{G_{i}}+\alpha_{v}\right) \sum_{h=1}^{i} a_{i h}^{4}+ \\
&+\left(\alpha_{v}^{2}+\alpha_{G_{i}}^{2}+2\left(\alpha_{v}+\alpha_{G_{i}}\right)\right)\left(6 \theta_{i}^{2} \sigma_{i}^{2}+3 \theta_{i}^{4}\right)+\quad \text { for } i=j=k=l \\
&+6\left[\left(\theta_{i}^{2}+\sigma_{i}^{2}\right)^{2} \alpha_{v} \alpha_{G_{i}}\right] \\
& k_{i i i l}= 3\left(\alpha_{v}^{2}+\alpha_{v}\right) \sum_{h=1}^{\min (i, l)} a_{i h}^{3} a_{l h}+\left(3 \alpha_{v}^{2}+6 \alpha_{v}\right) \theta_{i}^{3} \theta_{l}+ \\
&+\left(\alpha_{v}^{2}+2 \alpha_{v}\right)\left(3 \theta_{i} \theta_{l} \sigma_{i}^{2}+3 \theta_{i}^{2} \sigma_{i l}\right)+ \\
&+3\left(\theta_{i}^{2}+\sigma_{i}^{2}\right)\left(\theta_{i} \theta_{l}+\sigma_{i l}\right) \alpha_{V} \alpha_{G_{i}} \\
& k_{i i l l}= 3\left(\alpha_{v}^{2}+\alpha_{v}\right) \sum_{h=1}^{\min (i, l)} a_{i h}^{2} a_{l h}^{2}+\left(3 \alpha_{v}^{2}+6 \alpha_{v}\right) \theta_{i}^{2} \theta_{l}^{2}+ \\
&+\left(\alpha_{v}^{2}+2 \alpha_{v}\right)\left(\theta_{l}^{2} \sigma_{i}^{2}+4 \theta_{i} \theta_{l} \sigma_{i l}+\theta_{i}^{2} \sigma_{l}^{2}\right)+ \\
&+\left(\theta_{i}^{2}+\sigma_{i}^{2}\right)\left(\theta_{l}^{2}+\sigma_{l}^{2}\right)\left(\alpha_{G_{i}} \alpha_{G_{l}}+\alpha_{G_{i}} \alpha_{v}+\alpha_{v} \alpha_{G_{l}}\right) \\
& k_{i i k l}= 3\left(\alpha_{v}^{2}+\alpha_{v}\right) \sum_{h=1}^{\min (i, k, l)} a_{i h}^{2} a_{k h} a_{l h}+\left(3 \alpha_{v}^{2}+6 \alpha_{v}\right) \theta_{i}^{2} \theta_{k} \theta_{l}+ \\
&+\left(\alpha_{v}^{2}+2 \alpha_{v}\right)\left(\theta_{k} \theta_{l} \sigma_{i}^{2}+2 \theta_{i} \theta_{l} \sigma_{i k}+2 \theta_{i} \theta_{k} \sigma_{i l}+\theta_{i}^{2} \sigma_{k l}\right)+\quad \text { for } i=j \neq k=l \\
&+\left(\theta_{i}^{2}+\sigma_{i}^{2}\right)\left(\theta_{k} \theta_{l}+\sigma_{k l}\right) \alpha_{V} \alpha_{G_{i}} \\
& k_{i, j, k, l}=3\left(\alpha_{v}^{2}+\alpha_{v}\right) \sum_{h=1}^{\min (i, j, k, l)} a_{i h} a_{j h} a_{k h} a_{l h}+\left(3 \alpha_{v}^{2}+6 \alpha_{v}\right) \theta_{i} \theta_{j} \theta_{k} \theta_{l}+ \\
&+\left(\alpha_{v}^{2}+2 \alpha_{v}\right)\left(\theta_{k} \theta_{l} \sigma_{i, j}+\theta_{i} \theta_{l} \sigma_{j k}\right) \\
&+\left(\alpha_{v}^{2}+2 \alpha_{v}\right)\left(\theta_{j} \theta_{l} \sigma_{i k}+\theta_{i} \theta_{k} \sigma_{j l}+\theta_{j} \theta_{k} \sigma_{i l}+\theta_{i} \theta_{j} \sigma_{k l}\right) \\
& \text { for } i \neq j \neq k \neq l
\end{aligned}
$$


The analytical expected utility function has the following form:

$$
\begin{aligned}
E\left[u\left(\sum_{i=1}^{N} w_{i} X_{i}\right)\right] & =E\left(-\exp \left(-\lambda \sum_{i=1}^{N} w_{i} X_{i}\right)\right) \\
& =-\exp \left(-\lambda \sum_{i=1}^{N} w_{i} \mu_{i}+\sum_{i=1}^{N} \alpha_{G_{i}} \ln \left(\frac{1}{1+\lambda w_{i} \theta_{i}-\frac{\lambda^{2}}{2} w_{i}^{2} \sigma_{G_{i^{2}}}}\right)\right) * \\
& * \exp \left(\alpha_{v} \ln \left(\frac{1}{1+\lambda \sum_{i=1}^{N} w_{i} \theta_{i}-\frac{\lambda^{2}}{2} \sum_{i, j=1}^{N} w_{i} w_{j} \sum_{h=1}^{\min (i, j)} a_{i h} a_{j h}}\right)\right)
\end{aligned}
$$

\section{Empirical Analysis}

In this section we perform an empirical analysis based on a portfolio composed of 18 assets taken from the SP 500 index. We have chosen the 9 most and the 9 less traded assets on $05 / 09 / 2011$. The dataset consists of 3045 daily return observations, from $01 / 04 / 2000$ to $01 / 09 / 2011$. We have used a rolling window strategy of 6 months in-sample and 2 months out-of-sample.

The first step is the in-sample parameter estimation for each MVG model. In tables 1, 2, 3, 4 and 5 we report the estimated parameters for the three MVG models and the first in-sample window.

Table [1, 2, 3, 4 and 5 here]

Once we have estimated the parameters, we calculate,for the in-sample window, moments and comoments for the sample approach and MVG models. Then, we find the in-sample optimal weights solving the optimization problem (4) for different levels of risk aversion. We have done this analysi for $' \lambda=5,10,20,30$ '. The optimization is performed through Matlab function FMINCON.

Finally, we find the optimal weights, for each in sample period, using no longer the Taylor expansion but using the analytical CARA expected utility function. As an example, we show in table 6 the optimal weights obtained using four moments portfolio optimization procedure using Wang model and $\lambda=30$.

Table [6] here

In order to have a diversified portfolio we require the lower bound, for weights, to be $2 * 10^{-4}$. Holding these weights constant for the next two months we calculate the out-of-sample returns, for each portfolio. The out-of-sample portfolio statistics are reported in table 7 .

Table [7] here 
For each level of risk aversion we have reported the percentage annual mean, percentage annual standard deviation, skewness and kurtosis of the out-ofsample portfolio returns. We remark that an investor prefers a portfolio with the highest mean and skewness and lowest standard deviation and kurtosis. For each level of risk aversion, in Table 7, we have used boldface to indicate the portfolios that have the highest mean and skewness and lowest standard deviation and kurtosis and, as we can observe, it is not easy to choose between the different portfolios because the portfolio with the highest mean is not the same portfolio that has the lowest standard deviation, and so on. Therefore observing these results we are unable to say if one model is better than another.

In order to select a model, we have decided to use the Monetary Utility Gain/Loss Measure (MUG), proposed by Ang and Bekaert (2002).

The MUG is obtained solving the following equation w.r.t $W_{a}$ :

$$
\frac{1}{T} \sum_{t=1}^{T}-e^{-\lambda\left(1+r_{t}^{(\text {model b })}\right)}=\frac{1}{T} \sum_{t=1}^{T}-e^{-\lambda W_{a}\left(1+r_{t}^{(\text {model } a)}\right)},
$$

where $r_{t}^{\text {(model a) }}\left(r_{t}^{\text {(model b) })}\right)$ are annualized geometric, out-of-sample, portfolio returns obtained using "model a" ("model b"). The percentage annual required payment (MUG of "model $b$ " with respect to "model $a$ ") is given by $M U G=\left(W_{a}-1\right) * 100$.

MUG represents the excess amount invested in the portfolio obtained using "model a" that gives the same terminal wealth achieved by investing one euro in the portfolio obtained with "model b". If $M U G>0$ we prefer "model b" instead of " model a" and vice-versa when $M U G<0$.

In Table 8 we report the percentage annual MUG of the different portfolios obtained solving the optimization problem (4).

\section{Table 8 here}

The column labeled "MVG versus sample $M V$ " shows that MVG models are preferred with respect to sample approach: the MUG is always positive independently from the MVG model used. For all levels of risk aversion, the highest MUG is the one obtained using Wang model, when we consider a Taylor expansion truncated at the 2 nd order.

In column labeled "MVG versus sample MVSK" the conclusions are the same as in the previous case, except for $\lambda=30$ where MUG is negative for the Semeraro model.

In column labeled "MVG MVSK versus $M V G M V$ " the model 1 and Wang model gives always a positive MUG, meaning that four moments portfolio allocation is better than two moments. When the Semeraro model is used, we have obtained unexpected results since the MUG is almost always negative. A possible explanation is that in this model there is only one parameter ' $n$ ' modeling the dependence structure between assets.

In the last column, "Sample \& versus sample 2 moments", there is the MUG obtained when we compare the sample portfolio allocation with four moments 
with the sample portfolio allocation with two moments. In this case, four moments portfolio allocation is better than the two moments portfolio allocation except for $\lambda=10$.

In table 9 we report the annual percentage MUG between the analytical CARA utility function and the multivariate variance gamma with 2 moments and 4 moments.

Table 9 here

Except for the Semeraro model, the 4th order Taylor expansion seems to approximate better than the 2nd order the MVG analytical function ; since the MUG reported in column labeled "MVG analytical versus $M V G M V S K$ " is almost always closer to zero than the MUG reported in column labeled " $M V G$ analytical versus $M V G M V$ ".

\section{Conclusions}

In this paper we have performed an out-of-sample, empirical analysis on a portfolio of 18 assets taken from S\&P500. In order to introduce higher moments in portfolio allocation we have considered the Taylor expansion of the utility function. For moments and comoments estimation we have used the sample approach and the multivariate variance gamma approach. The measure used to compare the different portfolios with each other is the MUG.

We find that a MVG model with two moments is better than the sample approach with two moments and it generates a significant improvement for investors and in particular the MVG model proposed by Wang generates higher improvement compared to the other two MVG models.

A MVG model with four moments is almost always better than the sample approach with four moments, because it gives a positive MUG.

When four moments and two moments portfolios are compared we have obtained that in general four moments generate a significant improvement for investors when the MVG 'model 1', 'Wang model' or the 'sample approach' are used. While when Semeraro model is used we have obtained that two moments portfolio allocation is better than the four moments one.

Using the analytical expected utility function we obtained that, in terms of MUG, four moments Taylor expansion approximates this function better than two moments Taylor expansion when the MVG 'model 1' or the 'Wang model' are used. This is not the case for Semeraro model. The unexpected results in case of Semeraro model are due perhaps to the fact that we have only one parameter that captures the dependence structure between assets. This model is easily implemented and in general it has less parameters to estimate compared with the other two MVG models, but having only one parameter that captures the dependence structure between assets is not enough, especially when the number of assets in portfolio is high.

The results obtained in this paper are important for portfolio managers who use portfolio optimization incorporating higher moments. 


\section{References}

[1] Ang, A., ANd BeKaert, G. International asset allocation with regime shifts. Review of Financial Studies 15, 4 (2002), 1137-1187.

[2] Athayde, G., and Flores, R. G. The portfolio frontier with higher moments: The undiscovered country. Computing in Economics and Finance 2002 209, Society for Computational Economics, 2002.

[3] Athayde, G., and Flores, R. G. On certain geometric aspects of portfolio optimisation with higher moments. In Multi-Moment Asset Allocation and Pricing Model, J. F. Jurczenko and B. B. Maillet, Eds. John Wiley \& Sons, 2006, pp. 37-50.

[4] Bertini, C., Lozza, S. O., and Staino, A. Discrete time portfolio selection with lévy processes. In Proceedings of the 8th international conference on Intelligent data engineering and automated learning (Berlin, Heidelberg, 2007), IDEAL'07, Springer-Verlag, pp. 1032-1041.

[5] Cont, R., and Tankov, P. Financial Modelling with Jump Processes. Chapman \& Hall/CRC Financial Mathematics Series, 2003.

[6] Desmoulins-Lebeault, F. Gram-charlier expansions and portfolio selection in non-gaussian universes. In Multi-Moment Asset Allocation and Pricing Model, J. F. Jurczenko and B. B. Maillet, Eds. John Wiley \& Sons, 2006, pp. 79-112.

[7] Hitaj, A., Martellini, L., and Zambruno, G. Optimal hedge fund allocation with improved estimates for coskewness and cokurtosis parameters. Techincal report. furthercoming on journal of alternative investments, EDHEC-Risk Institute, 2010.

[8] JEAn, W. H. More on multidimensional portfolio analysis. Journal of Financial and Quantitative Analysis 8, 03 (1973), 475-490.

[9] Jondeau, E., Poon, S.-H., And Rockinger, M. Financial modeling under non-gaussian distributions. Springer Finance. Springer, 2007.

[10] Jondeau, E., And Rockinger, M. Optimal portfolio allocation under higher moments. European Financial Management 12, 01 (2006), 29-55.

[11] LoistL, O. The erroneous approximation of expected utility by means of a taylor's series expansion: Analytic and computational results. American Economic Review 66, 5 (1976), 904-10.

[12] Loregian, A., Mercuri, L., And Rroji, E. Approximation of the variance gamma model with a finite mixture of normals. Statistics 83 Probability Letters 82, 2 (2011), 217 - 224.

[13] Madan, D. B., And Seneta, E. The variance-gamma (v. g.) model for share market returns. Journal of Business 63, 4 (1990), 511-524. 
[14] Madan, D. B., And Yen, J. J. Chapter 23 asset allocation with multivariate non-gaussian returns. In Financial Engineering, J. R. Birge and V. Linetsky, Eds., vol. 15 of Handbooks in Operations Research and Management Science. Elsevier, 2007, pp. 949 - 969.

[15] Markowitz, H. Portfolio selection. The Journal of Finance 7, 1 (1952), 77-91.

[16] Martellini, L., And Ziemann, V. Improved estimates of higher-order comoments and implications for portfolio selection. Review of Financial Studies 23, 4 (2010), 1467-1502.

[17] Meucci, A. Risk and Asset Allocation. Springer Finance. Springer, 2005.

[18] Rubinstein, M. E. The fundamental theorem of parameter-preference security valuation. Journal of Financial and Quantitative Analysis 8, 01 (1973), 61-69.

[19] Semeraro, P. A multivariate variance gamma model for financial applications. International Journal of Theoretical and Applied Finance (IJTAF) 11, 01 (2008), 1-18.

[20] Wang, J. The Multivariate Variance Gamma Process and its Applications in Multi-asset Option Pricing. PhD thesis, Faculty of the Graduate School of the University of Maryland, 2009. 


\begin{tabular}{|c|c|c|c|}
\hline \multicolumn{4}{|c|}{ Estimated parameters for the first model } \\
\hline \hline Ticker & $\mu_{0}$ & $\theta$ & $\alpha$ \\
\hline A UN Equity & -0.0077 & 0.0075 & 1.1831 \\
AA UN Equity & -0.0132 & 0.0093 & 1.1831 \\
AAPL UW Equity & -0.0197 & 0.0166 & 1.1831 \\
ABC UN Equity & -0.0058 & 0.0101 & 1.1831 \\
ABT UN Equity & -0.0074 & 0.0081 & 1.1831 \\
ACE UN Equity & -0.0263 & 0.0267 & 1.1831 \\
ADBE UW Equity & -0.0024 & 0.0071 & 1.1831 \\
ADI UN Equity & -0.0234 & 0.0238 & 1.1831 \\
ADM UN Equity & -0.0046 & 0.0029 & 1.1831 \\
ZION UW Equity & 0.0029 & -0.0034 & 1.1831 \\
YUM UN Equity & -0.0057 & 0.0033 & 1.1831 \\
YHOO UW Equity & -0.0032 & -0.0009 & 1.1831 \\
XRX UN Equity & -0.0062 & 0.0048 & 1.1831 \\
XRAY UW Equity & -0.0008 & 0.0024 & 1.1831 \\
XOM UN Equity & -0.0119 & 0.0104 & 1.1831 \\
XLNX UW Equity & 0.0083 & -0.0029 & 1.1831 \\
XL UN Equity & -0.0038 & 0.0041 & 1.1831 \\
XEL UN Equity & -0.0035 & 0.0034 & 1.1831 \\
\hline
\end{tabular}

Table 1: Estimated parameters for the first model in first calibration window.

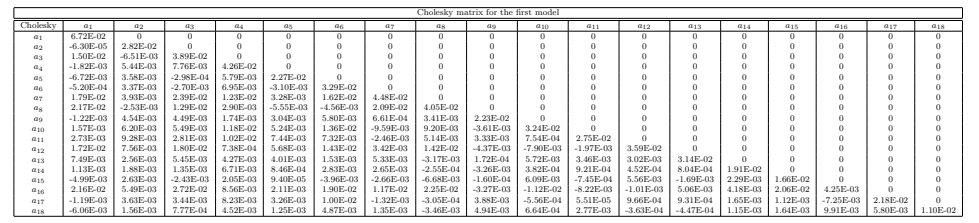

Table 2: Estimated Cholesky matrix for Model 1 in first calibration period.

\begin{tabular}{|c|c|c|c|c|c|}
\hline \multicolumn{7}{|c|}{ Estimated parameters for Semeraro model } \\
\hline \hline Ticker & $\mu_{0, i}$ & $\theta_{i}$ & $\sigma_{i}$ & $\mathrm{l}$ all & $\mathrm{n}$ all \\
\hline A UN Equity & -0.0076 & 0.0076 & 0.0671 & 0.2140 & 0.9260 \\
AA UN Equity & -0.0129 & 0.0089 & 0.0282 & 0.2846 & 0.9260 \\
AAPL UW Equity & -0.0170 & 0.0124 & 0.0430 & 0.5207 & 0.9260 \\
ABC UN Equity & -0.0056 & 0.0108 & 0.0435 & 0.1396 & 0.9260 \\
ABT UN Equity & -0.0072 & 0.0077 & 0.0246 & 0.3058 & 0.9260 \\
ACE UN Equity & -0.0265 & 0.0272 & 0.0338 & 0.2366 & 0.9260 \\
ADBE UW Equity & -0.0023 & 0.0069 & 0.0578 & 0.2824 & 0.9260 \\
ADI UN Equity & -0.0167 & 0.0160 & 0.0537 & 0.4572 & 0.9260 \\
ADM UN Equity & -0.0047 & 0.0031 & 0.0244 & 0.1719 & 0.9260 \\
ZION UW Equity & 0.0027 & -0.0038 & 0.0407 & 0.0359 & 0.9260 \\
YUM UN Equity & -0.0057 & 0.0034 & 0.0334 & 0.1584 & 0.9260 \\
YHOO UW Equity & -0.0035 & -0.0006 & 0.0500 & 0.3743 & 0.9260 \\
XRX UN Equity & -0.0065 & 0.0062 & 0.0348 & 0.0000 & 0.9260 \\
XRAY UW Equity & -0.0008 & 0.0024 & 0.0211 & 0.2576 & 0.9260 \\
XOM UN Equity & -0.0106 & 0.0085 & 0.0219 & 0.3901 & 0.9260 \\
XLNX UW Equity & 0.0082 & -0.0026 & 0.0549 & 0.4064 & 0.9260 \\
XL UN Equity & -0.0039 & 0.0045 & 0.0275 & 0.1487 & 0.9260 \\
XEL UN Equity & -0.0035 & 0.0034 & 0.0197 & 0.2446 & 0.9260 \\
\hline
\end{tabular}

Table 3: Estimated parameters for Semeraro model in first calibration period. 


\begin{tabular}{|c|c|c|c|c|}
\hline \multicolumn{6}{|c|}{ Estimated parameters for Wang model } \\
\hline \hline Ticker & $\alpha_{g_{i}}$ & $\alpha_{v}$ & $\mu_{0, i}$ & $\theta_{i}$ \\
\hline A UN Equity & 0.2140 & 0.9260 & -0.0076 & 0.0076 \\
AA UN Equity & 0.2846 & 0.9260 & -0.0129 & 0.0089 \\
AAPL UW Equity & 0.5207 & 0.9260 & -0.0170 & 0.0124 \\
ABC UN Equity & 0.1396 & 0.9260 & -0.0056 & 0.0108 \\
ABT UN Equity & 0.3058 & 0.9260 & -0.0072 & 0.0077 \\
ACE UN Equity & 0.2366 & 0.9260 & -0.0265 & 0.0272 \\
ADBE UW Equity & 0.2824 & 0.9260 & -0.0023 & 0.0069 \\
ADI UN Equity & 0.4572 & 0.9260 & -0.0167 & 0.0160 \\
ADM UN Equity & 0.1719 & 0.9260 & -0.0047 & 0.0031 \\
ZION UW Equity & 0.0359 & 0.9260 & 0.0027 & -0.0038 \\
YUM UN Equity & 0.1584 & 0.9260 & -0.0057 & 0.0034 \\
YHOO UW Equity & 0.3743 & 0.9260 & -0.0035 & -0.0006 \\
XRX UN Equity & 0.0000 & 0.9260 & -0.0065 & 0.0062 \\
XRAY UW Equity & 0.2576 & 0.9260 & -0.0008 & 0.0024 \\
XOM UN Equity & 0.3901 & 0.9260 & -0.0106 & 0.0085 \\
XLNX UW Equity & 0.4064 & 0.9260 & 0.0082 & -0.0026 \\
XL UN Equity & 0.1487 & 0.9260 & -0.0039 & 0.0045 \\
XEL UN Equity & 0.2446 & 0.9260 & -0.0035 & 0.0034 \\
\hline
\end{tabular}

Table 4: Estimated parameters in first calibration window for Wang model.

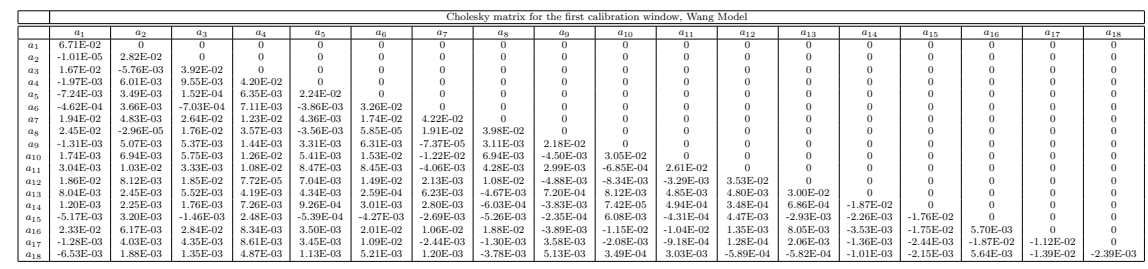

Table 5: Estimated Cholesky matrix for Wang model in first calibration period. 


\begin{tabular}{|c|c|c|c|c|c|c|c|c|c|c|c|c|c|c|c|c|c|}
\hline & & & & & & & & & & & & & & & & & \\
\hline & & 0.000 & $\frac{0.0}{0.0}$ & & & & $\begin{array}{l}\text { EFaniti } \\
0.0 .0641\end{array}$ & & & & & & & & 060 & $\frac{4}{101010}$ & \\
\hline & 0 & $\begin{array}{l}0.000 \\
0.00 \\
0.00\end{array}$ & 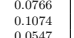 & 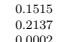 & $\begin{array}{l}0.08 \\
0.06\end{array}$ & 1098 & $\begin{array}{l}0.040990 \\
0.00002\end{array}$ & & & $\begin{array}{l}0.0 .00 \\
0.00\end{array}$ & & $\begin{array}{l}0.0 .0 \\
0.08\end{array}$ & & & $\begin{array}{l}0.00002 \\
0.0002\end{array}$ & 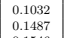 & .0738 \\
\hline & 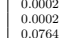 & & & 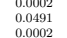 & $\begin{array}{l}0.0 \\
0.0 \\
0.0\end{array}$ & & & & & & & & & & & & \\
\hline & 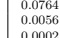 & $\begin{array}{l}0.10 \\
0.11 \\
0.00\end{array}$ & & $\begin{array}{c}0.001 \\
0.01 \\
0.00\end{array}$ & $\begin{array}{l}0.0 \\
0.0 \\
.00\end{array}$ & & & & & & & & & & & & $\begin{array}{l}0.41969 \\
0.4\end{array}$ \\
\hline & $\begin{array}{l}1.0002 \\
.0002 \\
\end{array}$ & $\begin{array}{l}x_{0.00}^{0.00} \\
0.00\end{array}$ & 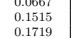 & $\begin{array}{l}.0 .051 \\
0.21 \\
0.15\end{array}$ & $\begin{array}{l}0.00 \\
0.00 \\
0.00\end{array}$ & $\begin{array}{l}\text { o. } 0.0727 \\
.00002\end{array}$ & $\begin{array}{l}\text { o. } \\
\text { o. } \\
0.000\end{array}$ & & & 40 & & & & & $\begin{array}{l}0.0 \\
0.0 \\
0.0\end{array}$ & 0.0 & \\
\hline & .0.0002 & $\begin{array}{l}\frac{0.020}{0.0220} \\
0.020\end{array}$ & $\begin{array}{l}0.082929 \\
0.15358\end{array}$ & $\begin{array}{l}0.072 \\
0.00 \\
0.00\end{array}$ & $\begin{array}{l}0.000 \\
0.000\end{array}$ & 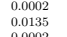 & $\begin{array}{l}\substack{0.000 \\
0.000 \\
0,000} \\
0\end{array}$ & 0.000 & & $\frac{0.4}{2}$ & & & & & $\begin{array}{l}0.0 .00 \\
0.00\end{array}$ & 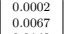 & $\begin{array}{l}0.0 \\
0.0 \\
0.0\end{array}$ \\
\hline & & $\begin{array}{l}0.0000 \\
0.000\end{array}$ & & $\begin{array}{l}0.0 .00 \\
0.00\end{array}$ & & & & & & & & & & & & & $\begin{array}{l}0.007 \\
0.0000\end{array}$ \\
\hline & $\begin{array}{c}0.000202 \\
.0002\end{array}$ & 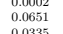 & $\begin{array}{l}0.00602 \\
0.0002 \\
0.0002\end{array}$ & 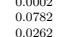 & 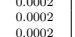 & $\begin{array}{l}0.00020 \\
0.0002 \\
0.0002\end{array}$ & $\mid \begin{array}{l}0.0000 \\
0.000 \\
0.000\end{array}$ & t. & & $\begin{array}{l}\text { o. oud } \\
\text { ood } \\
\text { out }\end{array}$ & & & & & $\begin{array}{l}0.00 \\
0.0 \\
0.0\end{array}$ & $\mid \begin{array}{l}0.07 \\
0.07 \\
0.1\end{array}$ & 0.0080 \\
\hline & .000 & $\begin{array}{l}0.0000 \\
0.0991\end{array}$ & $\begin{array}{l}0.0002 \\
0.0971\end{array}$ & & & & & & & & & & & & & & 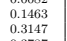 \\
\hline & $\begin{array}{l}0.0002 \\
0.0490 \\
0\end{array}$ & $\begin{array}{l}0.11777 \\
0.0557\end{array}$ & $\begin{array}{l}\substack{0.0002 \\
0.0002} \\
0.0002\end{array}$ & $\begin{array}{l}0.000 \\
0.000 \\
0.000\end{array}$ & $\begin{array}{l}0.0000 \\
0.000 \\
0.00\end{array}$ & $\begin{array}{l}\frac{0.000}{0.0000} \\
\text { o. }\end{array}$ & 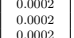 & 1.3434 & & $\begin{array}{l}0.08 \\
\text { o.4. } \\
\text { and }\end{array}$ & & & & & & & \\
\hline & $\begin{array}{l}.0337 \\
0.0002\end{array}$ & $\begin{array}{l}\text { 然 } 00002 \\
0.0002\end{array}$ & $\begin{array}{l}0.000 \\
0.0000 \\
0.040\end{array}$ & $\begin{array}{l}0.00 \\
0.00 \\
0.00\end{array}$ & $\begin{array}{l}0.25 \\
0.22 \\
0.11\end{array}$ & & {$\left[\begin{array}{l}0.00 \\
0\end{array}\right.$} & & & & & & & & & & \\
\hline & $0_{000}^{00}$ & $\begin{array}{l}0.1481 \\
0.12891\end{array}$ & $\begin{array}{l}0.000202 \\
0.0002\end{array}$ & $\begin{array}{l}0.0 .0 \\
0.07\end{array}$ & $\begin{array}{l}0.000 \\
0.000\end{array}$ & $\begin{array}{l}.0 .05 \\
0.10\end{array}$ & 0.00 & & & & & & & & & $\begin{array}{l}0.000 \\
0.000\end{array}$ & \\
\hline & .0.002 & 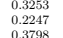 & $\begin{array}{l}0.00020 \\
0.0002 \\
0.0002\end{array}$ & $\begin{array}{l}.0 .000 \\
0.000 \\
0.00\end{array}$ & $\begin{array}{l}0.000 \\
0.000 \\
0.00\end{array}$ & $\begin{array}{l}0.05 \\
0.000 \\
0.000\end{array}$ & $\begin{array}{l}0.000 \\
0.00 \\
0.00\end{array}$ & 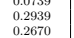 & & & & & & & & & $\begin{array}{l}0.000 \\
0.002 \\
0.000\end{array}$ \\
\hline & $\begin{array}{l}0.00 \\
0.000 \\
0.00\end{array}$ & $\begin{array}{l}\text { o. } 0.0711 \\
0.00100\end{array}$ & $\begin{array}{l}0.00963 \\
0.010682\end{array}$ & $\begin{array}{l}\text { o. } 0.344 \\
0.1939\end{array}$ & i.0.0 & $\begin{array}{l}0.000 \\
0.0000 \\
0.000\end{array}$ & $\begin{array}{l}0.00 \\
0.00 \\
0.00\end{array}$ & $\begin{array}{l}0.000 \\
0.00\end{array}$ & & & & & & & & & \\
\hline .26 & 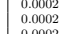 & 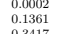 & $\begin{array}{l}0.0 .30366 \\
0.3341\end{array}$ & $\begin{array}{l}.0 .00 \\
0.00 \\
0.00\end{array}$ & $\begin{array}{l}0.003 \\
0.08\end{array}$ & $\begin{array}{l}0.000 \\
0.000\end{array}$ & $\begin{array}{l}0.000 \\
0.000\end{array}$ & & & & & & & & & & $\begin{array}{l}0.315 \\
0.000 \\
0.000\end{array}$ \\
\hline & $\begin{array}{l}1.0002 \\
.02227 \\
\end{array}$ & 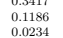 & 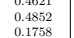 & $\begin{array}{l}\text { o. } \\
0.000 \\
0.000\end{array}$ & $\begin{array}{l}.0 .07 \\
0.07 \\
0.007\end{array}$ & $\begin{array}{l}0.08 \\
0.012 \\
0.08\end{array}$ & 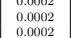 & 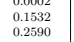 & & & & & & & & & \\
\hline & $\begin{array}{l}0.000 \\
0.000 \\
0.000\end{array}$ & $\begin{array}{l}0.0002 \\
0.00002\end{array}$ & wower & $c^{0.22}$ & & & \begin{tabular}{|l}
0.00 \\
0
\end{tabular} & & & & & & & & & & \\
\hline 1006 & .0002 & $\begin{array}{l}0.0129 \\
0.1375\end{array}$ & 0.027 & & & & $\begin{array}{l}0.00 \\
0.00\end{array}$ & & & & & & & & & & \\
\hline & 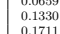 & 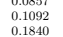 & $\begin{array}{l}0.000002 \\
0.0034\end{array}$ & $\begin{array}{l}0.366 \\
0.369 \\
0.090\end{array}$ & $\begin{array}{l}0.00 \\
0.00 \\
0.00\end{array}$ & $\begin{array}{l}0.000 \\
0.00 \\
0.00\end{array}$ & $\begin{array}{l}0.03 \\
0.13 \\
0.00\end{array}$ & o.ood & & & & & & & & & \\
\hline & & 0.25 & & & & & $\begin{array}{l}0.00 \\
0.00\end{array}$ & & & & & & & & & & \\
\hline & 0.00000 & 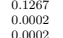 & 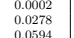 & $\begin{array}{l}0.12 \\
0.40 \\
0.02\end{array}$ & $\begin{array}{l}0.000 \\
0.00\end{array}$ & $\begin{array}{l}0.060 \\
0.000 \\
0.00\end{array}$ & 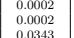 & . & & & & & & & & & \\
\hline & .0002 & 0.000 & & $\begin{array}{l}0.327 \\
0.027\end{array}$ & & & & & & & & & & & & & \\
\hline & .0.000 & $\begin{array}{l}0.0002 \\
0.00002\end{array}$ & $\begin{array}{l}0.0002 \\
0.07878\end{array}$ & $\begin{array}{l}.0 .72 \\
0.62\end{array}$ & $\begin{array}{l}0.000 \\
0.000\end{array}$ & $\begin{array}{l}0.00 \\
0.00\end{array}$ & 先, & & & & & & & & & & \\
\hline & $\begin{array}{l}1.0002 \\
.0002\end{array}$ & 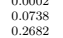 & $\begin{array}{l}0.060 \\
0.2060 \\
0.1545\end{array}$ & $\begin{array}{l}.0 .03 \\
0.03 \\
0.010\end{array}$ & & & & & & & & & & & & & \\
\hline & $\begin{array}{l}0.0 .002 \\
0.0002\end{array}$ & $\begin{array}{l}0.31966 \\
0.393640\end{array}$ & $\begin{array}{l}0.274 \\
0.223\end{array}$ & $\begin{array}{l}0.0008 \\
0.344\end{array}$ & $\begin{array}{l}0.000 \\
0.000\end{array}$ & $\begin{array}{l}0.0 \\
0.0\end{array}$ & \begin{tabular}{|l|}
0.11 \\
0.03
\end{tabular} \mid & $\begin{array}{l}0.0 . \\
0.0\end{array}$ & & & & $\begin{array}{l}0.0 . \\
0.0 .\end{array}$ & & & & & ar \\
\hline 0.27 & $\begin{array}{l}\text {.0.0002 } \\
\text {.0.002 }\end{array}$ & 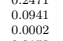 & 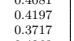 & 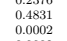 & 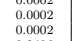 & 0.00 & 然.0. & $\begin{array}{l}0.0 \\
0.0 \\
0.0\end{array}$ & & & & $\begin{array}{l}0.0 .0 \\
0.0 .1 \\
0.1\end{array}$ & & & & & i. \\
\hline & & & & & & & & & & & & & & & & & \\
\hline & & & & & & & & & & & & & & & & & \\
\hline
\end{tabular}

Table 6: Optimal weights using Wang with four moments, $\lambda=30$ and lower bound 0.0002 . 


\begin{tabular}{|c|c|c|c|c|c|c|c|c|}
\hline \multicolumn{9}{|c|}{ Out-of-sample statistics } \\
\hline $\begin{array}{ll}\text { lambda } \\
\end{array}$ & \multicolumn{4}{|c|}{ lambda $=5$} & \multicolumn{4}{|c|}{ lambda $=10$} \\
\hline & annual mean (perc) & annual std (perc) & skewness & kurtosis & annual mean & annual std & skewness & kurtosis \\
\hline sample MV & 4.1376 & 26.2905 & -0.2365 & 8.1569 & 2.6706 & 22.3884 & -0.1540 & 7.3966 \\
\hline MVG mod 1 MV & 4.4233 & 25.7866 & -0.1775 & 7.8850 & 2.5264 & 21.6813 & -0.1148 & 7.1231 \\
\hline MVG sem MV & 4.0343 & 25.7745 & -0.0832 & 6.6688 & 3.9304 & 22.5615 & -0.0486 & 6.9731 \\
\hline MVG Wang MV & 4.5668 & 25.5352 & -0.1036 & 6.9431 & 3.3196 & 21.5800 & -0.0870 & 6.8265 \\
\hline sample MVSK & 4.4283 & 26.4425 & -0.2146 & 8.1439 & 2.6593 & 22.4086 & -0.1511 & 7.2766 \\
\hline MVG $\bmod 1 \mathrm{MVSK}$ & 4.7276 & 25.9006 & -0.1593 & 7.6018 & 2.9231 & 21.7740 & -0.1032 & 6.9489 \\
\hline MVG sem MVSK & 4.1173 & 25.8141 & -0.0811 & 6.6255 & 4.0407 & 22.6261 & -0.0456 & 6.9263 \\
\hline MVG Wang MVSK & 4.8639 & 25.6114 & -0.0927 & 6.8208 & 3.6848 & 21.6714 & -0.0784 & 6.7186 \\
\hline analytical mod 1 & 4.7868 & 25.8665 & -0.1599 & 7.6116 & 2.8394 & 21.7182 & -0.1035 & 6.9561 \\
\hline analytical sem & 4.0897 & 25.8113 & -0.0810 & 6.6247 & 4.0378 & 22.6171 & -0.0457 & 6.9315 \\
\hline analytical wang & 4.8356 & 25.5901 & -0.0930 & 6.8225 & 3.6312 & 21.6371 & -0.0790 & 6.7238 \\
\hline & \multicolumn{4}{|c|}{ lambda $=20$} & \multicolumn{4}{|c|}{ lambda $=$} \\
\hline lambda & annual mean (perc) & annual std (perc) & skewness & kurtosis & annual mean & annual std & skewness & kurtosis \\
\hline sample M & 1.5503 & 18.8626 & -0.1692 & 7.3830 & 1.2722 & 17.6454 & -0.1661 & 7.8709 \\
\hline MVG mod $1 \mathrm{MV}$ & & 18.5189 & -01664 & 74615 & 13866 & 17.4078 & -01677 & 80030 \\
\hline MVG sem MV & 3.0 & 19.6281 & -0.0659 & 7.4656 & 3.0192 & 18.4290 & -0.0728 & 7.9040 \\
\hline MVG Wang MV & 03 & 18.8626 & -0.1692 & 7.3830 & $\begin{array}{l}1.2722 \\
-132\end{array}$ & 17.6454 & -0.1661 & 7.8709 \\
\hline sampl & 1.4973 & 18.7951 & -0.1717 & 7.3351 & 1.3787 & 17.4848 & -0.1551 & 7.8194 \\
\hline MVG $\bmod 1 \mathrm{MVSK}$ & 1.9694 & 18.5883 & -0.1644 & $7.336 \mathrm{~s}-2>3$ & 1.7975 & 17.4464 & -0.1684 & 7.8608 \\
\hline MVG se & 3.2027 & 19.6701 & -0.0642 & 7.3953 & 3.1060 & 18.4579 & -0.0730 & 7.8335 \\
\hline MVG Wang MVSK & 2.8250 & 18.4520 & -0.1372 & 7.2088 & 2.6322 & 17.2994 & -0.1282 & 7.6145 \\
\hline analy & 1.9260 & 18.4928 & -0.1626 & 7.3748 & 1.7469 & 17.3096 & -0.1710 & 7.9533 \\
\hline analy & 3.2031 & 19.6390 & -0.0639 & 7.4034 & 3.1460 & 18.4156 & -0.0734 & 7.8529 \\
\hline analytical wang & 2.8493 & 18.3778 & -0.1344 & 7.2429 & 2.7731 & 17.2025 & -0.1236 & 7.6746 \\
\hline
\end{tabular}

Table 7: For different models, approaches and risk aversion we report out-ofsample portfolio statistics. The term 'MV' ('MVSK') means that we have used Taylor expansion truncated at the second (fourth) order. The term 'analytical' means that we have used the analytical expected utility function for portfolio allocation.

\begin{tabular}{|c|c|c|c|c|c|c|c|c|c|c|}
\hline \multicolumn{11}{|c|}{ Percentage annual MUG for different levels of risk } \\
\hline & $\overline{\text { MVG }}$ & ersus sampl & $\overline{\mathrm{MV}}$ & $\overline{\text { MVG v }}$ & sus sample & IVSK & MVG MY & K versus & $\overline{\mathrm{VG} \mathrm{MV}}$ & sample MVSK versus sample MV \\
\hline lambda & Model 1 & Semeraro & Wang & Model 1 & Semeraro & Wang & Model 1 & Semeraro & Wang & sample \\
\hline 5 & 1.0202 & 1.1851 & 1.8134 & 1.1436 & 1.1229 & 1.9686 & 0.1774 & 0.0297 & 0.1912 & 0.0886 \\
\hline 10 & 1.5625 & 1.9688 & 2.6906 & 1.8235 & 2.0367 & 2.8964 & 01986 & -00347 & 01643 & -0.0429 \\
\hline 20 & 1.3789 & 0.7442 & 3.0086 & 1.2536 & 0.5411 & 2.8387 & 0.0936 & -0.0513 & 0.0284 & 0.2431 \\
\hline 30 & 1.3450 & 0.8102 & 3.4680 & 0.4566 & -0.3752 & 2.5792 & 0.2026 & -0.0784 & 0.1707 & $\begin{array}{l}0.1829 \\
1.1829\end{array}$ \\
\hline
\end{tabular}

Table 8: The column labeled 'MVG versus sample MV' presents the MUG obtained when we consider one of the MVG models versus sample approach in 2nd-order expansion for expected utility. The column labeled 'MVG versus sample MVSK' reports the MUG obtained when we use one of the MVG models versus sample approach in 4nd-order expansion for expected utility. The column labeled 'MVG MVSK versus MVG MV' reports the MUG obtained when we use 4nd-order versus 2nd-order expansion for expected utility using one of the MVG models. The column labeled 'sample MVSK versus sample MV' reports the MUG obtained when we use 4nd-order versus 2nd-order expansion for expected utility using sample approach.

\begin{tabular}{|c|c|c|c|c|c|c|}
\hline \multicolumn{7}{|c|}{ Percentage annual analytical MUG for different levels of risk } \\
\hline \hline & \multicolumn{2}{|c|}{ MVG analytical versus MVG MV } & \multicolumn{3}{|c|}{ MVG analytical versus MVG MVSK } \\
\hline lambda & Model 1 & Semeraro & Wang & Model 1 & Semeraro & Wang \\
\hline 5 & 0.2764 & 0.0075 & 0.1907 & 0.0993 & -0.0222 & -0.0005 \\
10 & 0.2354 & -0.0185 & 0.1834 & 0.0360 & 0.0162 & 0.0191 \\
20 & 0.3992 & 0.0668 & 0.3222 & 0.3066 & 0.1178 & 0.2922 \\
30 & 0.8396 & 0.1812 & 0.8332 & 0.6354 & 0.2599 & 0.6474 \\
\hline
\end{tabular}

Table 9: For each MVG model, we report the MUG obtained using the analytical versus 2nd ('MVG analytical versus MV') and 4nd ('MVG analytical versus MVSK') order expansion for expected utility. 